\title{
A STUDY ON PRODUCT OPTIMIZATION DESIGN BASED ON GENETIC ALGORITHMS
}

\author{
Guiqin $\mathrm{Li}^{1}{ }^{1}$, Xiaojian Liu ${ }^{2}$, Qinfeng Yuan ${ }^{1}$, Minglun Fang ${ }^{1}$ \\ ${ }^{\prime}$ CIMS \& Robot Center of Shanghai University, Shanghai University, China; Email: \\ leeching@staff.shueducn. ${ }^{2}$ Industrial Design Institute, Northwestern Polytechnical \\ University, China.
}

Abstract: Genetic algorithms (GAs) can be viewed as random searching algorithm that based on the ideas of natural evolution. It is quite suitable for the complicated non-structural problems, for it has some properties such as self-adaptability, parallelity, evaluation-based, probability and multi-solution. Its principle is similar to human designers' behavior, a trial-and-error process guided by the evaluation regarding the goal: product optimization schemes. The paper analyzes the comparability between product optimization design and GAs, and proposes a technical method with its algorithm structures based on GAs. The codified representation, evaluation function and optimize searching of product design was basically realized based on GAs.

Key words: Product Optimization Design, Genetic algorithms, codified representation.

\section{INTRODUCTION}

General evolution theory works up from the investigation of artificial intelligence and cognition science. It transposes the notions of evolution in Nature to complex social system, AI system and artificial product system. Evolving optimization design method based on GAs gained new optimum product plan through inheriting and improving successful design cases.

Evolution theory and GAs have got many achievements in the domain of products design in recent years. For example, M. A. Rosenman in Sydney University developed a non-routine design system. ${ }^{[1]}$ The automatization department in Tsinghua University investigated soft-hard model based on generalized evolution. ${ }^{[2]}$ Literature [3] gave an evolution model of requirements analysis. The study of J. Poon and M. L. Maher applied

This project is supported by the National Natural Science Foundation of China under the grant No.50375090, the Scientific Research Fund of Shanghai Education Commotion under the grant No.202624 and the Shanghai University Science and Technology Development Fund of China under the Grant No.202615.

Please use the following format when citing this chapter:

Li, Guiqin, Liu, Xiaojian, Yuan, Qinfeng, Fang, Minglun, 2006, in International Federation for Information Processing (IFIP), Volume 207, Knowledge Enterprise: Intelligent Strategies In Product Design, Manufacturing, and Management, eds. K. Wang, Kovacs G., Wozny M., Fang M., (Boston: Springer), pp. 159-164. 
evolving principle to dynamic solution, taking into account the interaction of multi-factors during design process. ${ }^{[4]}$ Literature [5] set up an evolution model for design thoughts.

This article analyses the implementation of product optimization design process in the design/manufacture integrative process based on GAs.

\section{ANALYSIS OF THE PROBLEM OF PRODUCT OPTIMIZATION DESIGN}

So far, many of the product design CAD method are mostly for generating shape or structure of products. It's far from enough for evaluation and automatic adjustment of a plan. This investigation provides solution to harmonize the relations among product design principles, diversification, and optimization and design freedom, and to deal with the fuzzy decisions problem, via systematic classification and formalization product structural optimization design problem.

The main task of formulization is construct reasonable coding scheme (also named "genotype") of design problem (also named as "represent type"), viz it should express all forms of availability design plans, and has feasibility of arithmetic operation. For example, we can treat with shape factors as follows:(1)classify factors according to the hierarchy and put them into difference design stages;(2)For the shape of certain rudiment required, increase fitness artificially in two aspects: the ratio of eliminate through selection or contest, and the offspring individual number. So it is a dominant force in evolving direction;(3)Deal with the authority of local code segment, to differentiate the relative importance;(4)Rigid shape restrictions are treated by Arithmetic;(5)Provide interactive interface of code forcible operation and plan artificial selection.

\section{THE CODIFIED REPRESENTATION AND FITNESS FUNCTION OF PRODUCT OPTIMIZATION DESIGN}

Firstly, it's necessary to define a solution space that contains all feasible solutions for the program as the search area. The solution space for GAs is constructed by the code of the target problem. To product, the code is a set of parameters that describe the product features and properties. Different parameters represent different products.

Fitness function is assessing rule that determines acceptance or rejection a design plan in throng of random generated. The structure of product function model includes three layers: physics function, physiology function 
and psychology function. Physics function used to accomplish input/output tasks of the substance, energy, information or data that were schemed out. Physiology function used to accomplish interactive tasks between enactment product and user on action, contact, language or sense. Psychology function indicates to users' sensation and cognition information, including aesthetics, semantics and individuation, etc. The work of constructing fitness function then bases on the quantity model of this three function constraints.

Product code is composed of parameters that denote its functions. The form of the product code is as following, in which $M$ is function, whose value describes the function, such as length, weight, etc.

$$
B=\left\{\begin{array}{lllll}
M_{0}, & M_{1}, & M_{2}, & \ldots & M_{n-1}
\end{array}\right\}, M_{i} \in\left[\begin{array}{lll}
M_{i 0} & , & M_{i 1}
\end{array}\right]
$$

All rational values of every $M$ composed the solution space of the product, in which the best one lies.

According to the design flow, the search is based on the evaluation of the product code. There are different way of evaluating, depends on developer's purpose, such as technical evaluation, economical evaluation, aesthetics evaluation, etc. We adopted value engineering as the main evaluation method. We can get the value coefficient of each function:

$$
Z I_{i}=\frac{M I_{i}}{S_{i}}
$$

where $M I$ is function coefficient, $\sum M I_{i}=1$, and $\mathrm{S}$ is cost coefficient, $\sum S_{i}=1$. The total value of the product is calculated as:

$$
Z=\sum Z I_{i} * M_{i s}
$$

where $\mathrm{M}_{i s}$ is the importance coefficient of function $\mathrm{MI}_{i,}$ and $\sum M_{i s}=1$.

The evaluation criterion discussed above is an integral standard, and is mostly often used. Sometimes, we can use different criterion to evaluate a product from several aspects, and the selection and decision work is left to the users. 


\section{REALIZATION OF EVOLVING OPTIMIZATION DESIGN ARITHMETIC}

The conceit of arithmetic about main evolving concept and behavior in evolving calculation as follows:

a. Population: representation a series of coding plan to latency solution set of design problem

Original population generated with two methods: random method and object method. In which object method generates population with some design expectation, or some successful design cases through coding, or the optimization plans gained from previous round evolving calculation.

b. Inherit: maintenance in offspring design plans of part properties that are of some robust adaptability individual of the population

Inherit operation carry through reproduction and crossover.

c. Mutation: local changing in the coding segments

Mutation is main source of creativity in evolving design arithmetic. We plan traverse three mutation manners: innovation, adjustment and slighter shift. Innovation mostly used in conceptual design by way of biggish extent random mutation. In order to maintain creative content not eliminate through selection or contest, we can lower fitness function value artificially. Adjustment is to obtain diversity and highly fit individual through local modify code value in miniature. Slighter shift is to observe design results by continuance mutation of code segments to a certain direction based on some special requirements.

d. Selection: determination the selection of parent individual, survival of offspring, and the individual replaced by offspring in population based on the value of fitness function.

The product optimization design based on Genetic Algorithms is illustrated in Fig.1. The product optimization process is composed of four steps, which correspond to the five sub-processes of GAs random search, evaluation, recombination / mutation, decoding and interactive selection. They are performed by ten basic operators as follows.

$$
F=(M, N, O, P, Q, R, S, T, U, V)
$$

where $\mathrm{M}-\mathrm{C}$ individual construction operator, $\mathrm{N}-$ - constraints generating operator, $\mathrm{O}-$ - population maintenance operator, $\mathrm{P}-$ individual evaluation operator, $\mathrm{Q}-\mathrm{-}$ selection operator, $\mathrm{R}-{ }_{-}$ recombination operator, $\mathrm{S}-$ mutation operator, $\mathrm{T}$ - optimization control operator, $\mathrm{U}-$ decoding operator, $\mathrm{V}-$ interactive selection operator. 


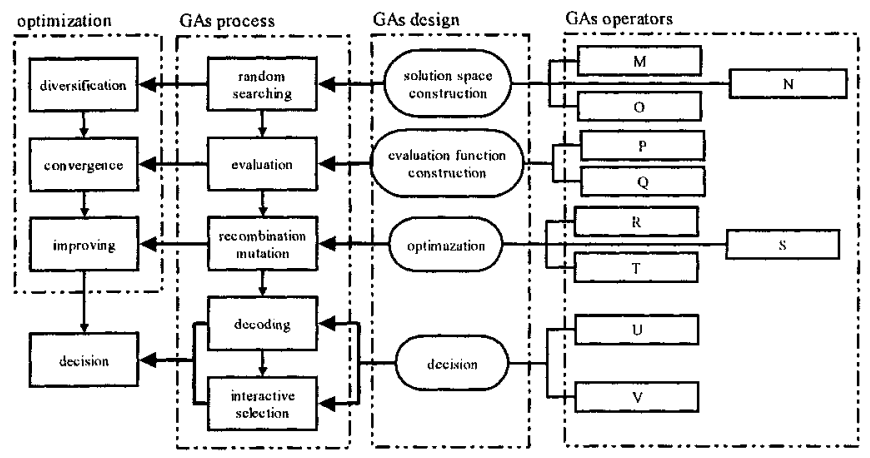

Figure 1. Product optimization design based on Genetic Algorithms

Fig.2 is the interface of the GAs based product design prototype we developed. Product optimization design flow can be divided into three stages: question conformation, automatic search and decision. Question conformation stage builds genotype and fitness function for design problem in interactive mode, and defines availability solution space. Automatic search stage is the operating process vs genotype in solution space. Decision stage is to select the ultimate plan through the interaction with user on the basis of work result, or decide next round search by user's determination till satisfied plan is obtained.

The system contains three functional parts: a) variable and parameter input, b) fitness function setting, c) search process setting. The design case in our prototype only take into account physics function, for other function is similarity. Computer is high efficient, but not versatile.

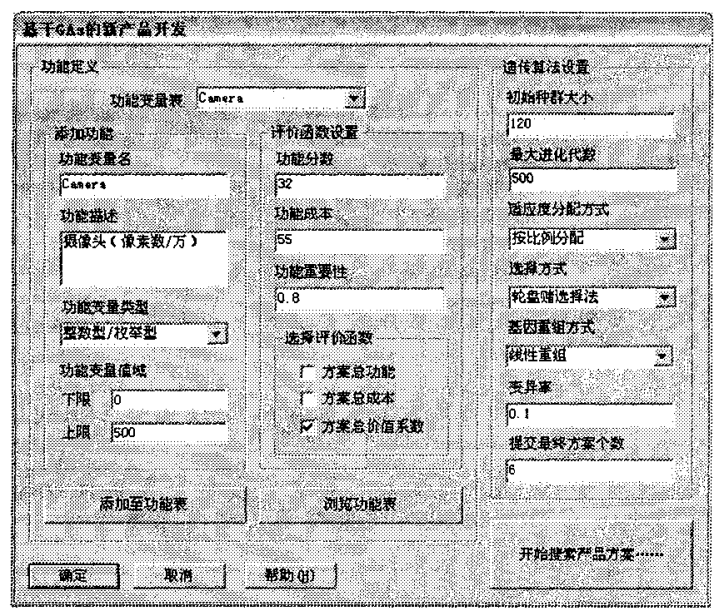

Figure 2. Interface of the GAs based product design system 


\section{CONCLUSION}

The GAs-based product optimization design makes use of the advantage of computer in speed, and realized basic intelligence in the evaluation of complex product plans. At the same time, it lefts a free space for designer's decision making. GAs is a useful mathematical tool to the simulation of human designer's design process guided by the evaluation regarding the goal. Function analysis is a proper way to code the product model. It well describes the product's function feature, and is convenient for the construction of fitness function GAs need.

\section{REFERENCES}

1. M. A. Rosenman, An exploration into evolutionary models for non-routine design, Artificial Intelligence in Engineering, 11(3), 287-293(1997).

2. ZHAO Nanyuan, Cognition Science and General Evolution Theory (Tsinghua University Press, Peking, 2002) (in Chinese).

3. CHENG Jingping, An investigation of demand analysis modeling for evolutionary product conceptual design, Hoisting and Conveying Machinery, (11), 6-8(2002).

4. J. Poon and M. L. Maher, Co-evolution and emergence in design, Artificial Intelligence in Engineering, 11(3). 319-327 (1997).

5. ZHANG Zhiwei, YE Qingtai, and WANG Anlin, Thought and systems of evolutionary deign, Journal of Shanghai Jiaotong University, 34(10), 1449-1452(2000) 